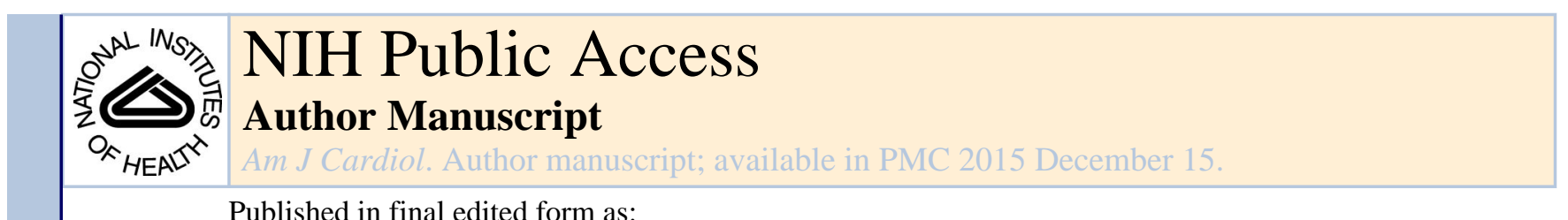

Published in final edited form as:

Am J Cardiol. 2014 December 15; 114(12): 1917-1922. doi:10.1016/j.amjcard.2014.09.034.

\title{
Relations between Depressive Symptoms, Anxiety, and T Wave Abnormalities in Subjects without Clinically-apparent Cardiovascular Disease (from the Multi-Ethnic Study of Atherosclerosis [MESA])
}

\author{
William Whang, MD, MS, James Peacock, MD, MS, Elsayed Z. Soliman, MD, MSc, MS, \\ Carmela Alcantara, PhD, Saman Nazarian, MD, Amit J. Shah, MD, Karina W. Davidson, \\ PhD, Steven Shea, MD, MS, Paul Muntner, PhD, and Daichi Shimbo, MD \\ Department of Medicine (W.W., J.P, C.A., K.W.D., S.S., D.S.), Columbia University, New York, \\ NY; Department of Epidemiology (S.S.), Mailman School of Public Health, Columbia University, \\ New York, NY; Epidemiological Cardiology Research Center (E.Z.S.), Department of \\ Epidemiology and Prevention, and Department of Medicine, Section on Cardiology, Wake Forest \\ School of Medicine, Winston Salem, NC; Department of Cardiology (S.N.), Johns Hopkins \\ University, Baltimore, MD; Department of Epidemiology (A.J.S.), Emory University, Atlanta, GA; \\ Department of Epidemiology (P.M.), University of Alabama at Birmingham
}

\begin{abstract}
We hypothesized that depression and anxiety were associated with electrocardiographic (ECG) repolarization abnormalities in the Multi-Ethnic Study of Atherosclerosis (MESA), a cohort free of symptomatic cardiovascular disease. Depressive symptoms were assessed by the Center for Epidemiologic Studies-Depression scale, and trait anxiety symptoms by the Spielberger trait anxiety scale; both were categorized according to uppermost quartile. T wave inversions in ECG leads other than V1-V3 were obtained from resting electrocardiograms at the baseline exam. Participants with major intraventricular conduction abnormalities, or taking anti-arrhythmics, antidepressants, and/or anti-psychotics were excluded. Logistic regression models were estimated with multivariable adjustment for traditional cardiovascular disease risk factors. Among 5,906 participants, elevated depressive symptoms were associated with increased odds of $\mathrm{T}$ wave inversions after multivariable adjustment (odds ratio $2.02,95 \%$ CI 1.33-3.06, $\mathrm{p}=0.001$ ) whereas greater trait anxiety was associated with a reduced odds of $\mathrm{T}$ wave inversions (odds ratio 0.47 , 95\% CI 0.29-0.77, $\mathrm{p}=0.003)$. The divergent associations of depressive symptoms and trait anxiety with ECG T wave inversions were similar in men and women, and these associations were present across the racial/ethnic subgroups (non-Hispanic white, African-American, Hispanic, Chinese). In conclusion, symptoms of depression and anxiety were independently, yet oppositely associated
\end{abstract}

(C) 2014 Elsevier Inc. All rights reserved.

Address for correspondence: William Whang, MD, MS, PH 9-321, 622 West $168^{\text {th }}$ Street, New York, NY 10032, Fax: 212-341-3431, Phone: 212-305-8620,ww42@cumc.columbia.edu.

Financial Conflicts of Interest: None

Publisher's Disclaimer: This is a PDF file of an unedited manuscript that has been accepted for publication. As a service to our customers we are providing this early version of the manuscript. The manuscript will undergo copyediting, typesetting, and review of the resulting proof before it is published in its final citable form. Please note that during the production process errors may be discovered which could affect the content, and all legal disclaimers that apply to the journal pertain. 
with ECG T wave inversions. Negative emotions may have differential impact on cardiovascular mortality through unique relations with cardiac repolarization.

\section{Keywords}

depression; anxiety; electrocardiogram; repolarization

\section{Introduction}

Cardiac repolarization measures by 12-lead electrocardiogram (ECG) have been recognized in population-based samples to be associated with cardiovascular mortality. ${ }^{1-4} \mathrm{~A}$ study of 10,899 Finnish middle-aged men and women without known heart disease found $\mathrm{T}$ wave inversions in ECG leads other than V1-V3 were associated with cardiac (relative risk 2.65, 95\% CI 1.86-3.78) and arrhythmic death (relative risk 3.16, 95\% CI 1.86-5.36) over an average follow-up of 30 years. ${ }^{4}$ Based on its prognostic utility, we examined the same indicator of abnormal repolarization to assess for its independent associations with depressive and anxiety symptoms in The Multi-Ethnic Study of Atherosclerosis (MESA). We hypothesized that depressive and anxiety symptoms would both be associated, albeit potentially differentially, with $\mathrm{T}$ wave abnormalities by ECG. We sought to identify a physiological measure that was related both with particular psychological symptoms and with cardiovascular mortality, such that it might provide insight into the mechanisms by which depression and anxiety are differentially related with cardiac mortality.

\section{Methods}

MESA is a multicenter, longitudinal cohort study of the prevalence, correlates, and progression of subclinical cardiovascular disease. ${ }^{5}$ MESA recruited 6,814 men and women 45 to 84 years old, self-identified as white, black, Hispanic, or Chinese and free of clinically apparent cardiovascular disease between July 2000 and August 2002 from six communities: Baltimore City and Baltimore County, Maryland; Chicago, Illinois; Forsyth County, North Carolina; Los Angeles County, California; northern Manhattan and Bronx, New York; and St. Paul, Minnesota. Informed consent was obtained from the participants and the study was approved by Institutional Review Boards of each participating center. To minimize potential secondary repolarization effects from organic heart disease, we excluded participants whose ECG had QRS duration $\geq 120 \mathrm{msec}$. Also, we excluded participants who reported taking anti-arrhythmic medications, anti-psychotic medications, and/or antidepressant medications.

Participants underwent an extensive baseline evaluation (Exam 1), including a standardized questionnaire, measurements of height, weight, body mass index, anthropometric data, and blood pressure, and blood samples for total and high-density lipoprotein cholesterol, triglycerides, and glucose levels after a 12 -hour fast. ${ }^{5}$ Information on demographics, current smoking, education level, current alcohol use, physical activity and medical history were obtained using standardized questionnaires. Education level was defined by the highest level of schooling achieved. Physical activity was defined as the total of all light, moderate, and vigorous activities multiplied by individual metabolic equivalent values (METS) for these activities. Diabetes was defined as a fasting serum glucose $\geq 126 \mathrm{mg} / \mathrm{dL}$ or use of 
hypoglycemic drugs or insulin. Serum creatinine was measured and estimated glomerular filtration rate (eGFR) was calculated by the Modification of Diet in Renal Disease equation. ${ }^{6}$ Urine albumin and creatinine were measured at the Clinical Chemistry Laboratory at Fletcher Allen Health Care (Burlington, Vermont). ${ }^{7}$ Urine albumin-tocreatinine ratios were classified according to presence of albuminuria ( $>30 \mathrm{mg}$ albumin/g creatinine). High sensitivity C-reactive protein was measured using a BNII nephelometer (Dade-Behring, Deerfield, Illinois). Cardiac magnetic resonance imaging (MRI) was performed with 1.5-T magnets with determination of left ventricular mass as described previously. ${ }^{8}$ Left ventricular mass index (LVMI) was calculated by using the formula LVMI=left ventricular mass/body surface area $(\mathrm{g} / \mathrm{m} 2)$.

Depressive symptoms were assessed at Exam 1 using the CES-D (Center for Epidemiologic Studies-Depression). ${ }^{9}$ The CES-D is a 20 -item survey that measures mood, somatic symptoms, social interactions, and motor functioning. Each item uses a a 4-point Likert scale for responses. The sum total over the 20 items can range from 0 to 60 , with higher scores indicating more severe symptoms of depression. The survey asks about the frequency in the past week that one has felt or behaved a certain way. Sample items include 'I felt depressed'; 'my sleep was restless'; 'I lost interest in my usual activities'. The CES-D does not include items for increased appetite or sleep, guilt, or suicidal thoughts. Anxiety was assessed at Exam 1 with the Trait-subscale of the State-Trait Anxiety Inventory. ${ }^{10}$ This is a 20-item scale that requires respondents to indicate how frequently they experience a variety of anxiety symptoms, also using a 4-point Likert scale. Based on the skewed distributions of both the CES-D scores and Trait Anxiety scores in the MESA sample (Supplemental Figure 1), we categorized elevated depressive symptoms and anxiety according to the upper quartile for each score. This resulted in a cut-point score of $\geq 10$ for the CES-D scale and $\geq 19$ for the Trait Anxiety scale. The cut-point of 10 for CES-D score has previously been used to define elevated depressive symptoms and has been associated with increased risk of ischemic heart disease events in a cohort without known cardiovascular disease. ${ }^{11,12}$

Standard 12-lead ECGs were digitally acquired at Exam 1 using a Marquette MAC-PC electrocardiograph (Marquette Electronics, Milwaukee, Wisconsin) at $10 \mathrm{~mm} / \mathrm{mV}$ calibration and speed of $25 \mathrm{~mm} / \mathrm{sec}$. All ECGs were centrally read at the EPICARE reading center, Wake Forest School of Medicine (Winston Salem, North Carolina), and visually inspected for technical errors and inadequate quality. The dependent variable for our analyses was presence of $\mathrm{T}$ wave inversions in leads other than $\mathrm{V} 1-\mathrm{V} 3$, according to the analysis by Aro et al. ${ }^{4}$ Specfically, this included any negative $\mathrm{T}$ wave amplitude $0.1 \mathrm{mV}$ or more in precordial leads V4, V5, or V6, or limb leads I, II, or aVF. T wave inversions in leads V1-V3 were not included in the dependent variable given their lack of prognostic significance. $^{4}$

Demographic and coronary disease risk factors for MESA participants included and excluded from the current analysis were compared using chi-square tests, analysis of variance, or non-parametric tests as appropriate. Among MESA participants included in our analysis, we compared these same characteristics across categories defined by the uppermost quartile of CES-D depressive symptom score and trait anxiety score. Logistic regression models were used to estimate the odds ratios for $\mathrm{T}$ wave inversions with elevated depressive 
symptoms and elevated anxiety, separately, with simultaneous inclusion of both symptom types. Two levels of adjustment were performed. First, we adjusted for age, gender, and race-ethnicity (White, African-American, Hispanic, Chinese). Next, odds ratios were calculated after further adjustment for center, body mass index, hypertension, diabetes mellitus, low-density lipoprotein (LDL) and high-density lipoprotein (HDL) cholesterol levels, heart rate on the resting ECG, systolic and diastolic blood pressure, cigarette smoking (never, former, or current), self-reported moderate and vigorous physical activity by quartile, estimated glomerular filtration rate, albuminuria, left ventricular hypertrophy according to Cornell ECG criteria, ${ }^{13}$ log-transformed highly-sensitive plasma C-reactive protein levels, benzodiazepine, and beta blocker use. Variance inflation factors were examined to evaluate the presence of multicollinearity. The consistency of the results were evaluated in subgroups defined by gender and race/ethnicity. Based on a prior analysis that observed a relationship between left ventricular cavity size and $\mathrm{T}$ wave amplitude, ${ }^{14}$ in a sensitivity analysis we estimated models that included left ventricular mass index measured by cardiac MRI as an additional covariable. All analyses were conducted using SPSS Version 22 (IBM Corp., Armonk, NY).

\section{Results}

After excluding participants with QRS duration $\geq 120 \mathrm{msec}$ and those taking anti-arrhythmic, antidepressant, and/or anti-psychotic medication, our sample consisted of 5,906 participants. Participants included in the analysis were younger (average age 62 years versus 63.2 years), less likely to be non-Hispanic white (35.6\% versus 56.9\%) and more likely ChineseAmerican, Hispanic, or African-American than participants who were excluded (Supplementary Table 1). Included participants had fewer coronary disease risk factors such as hypertension ( $44.2 \%$ versus $49.3 \%$ ) and current smoking (12.9\% versus $14.3 \%)$, and lower prevalence of reduced glomerular filtration rate $<60 \mathrm{ml} / \mathrm{min} / 1.73 \mathrm{~m}^{2}(9.1 \%$ versus $12.3 \%)$. Depressive and anxiety symptom scores were lower in the included sample.

Depressive symptom scores and anxiety scores were moderately correlated with each other (Spearman correlation $=0.48, \mathrm{p}<0.01$ ). Compared to participants with depressive symptoms score $<10$, those with elevated depressive symptoms were younger, more likely to be female, more frequently Hispanic, and more likely to report current smoking (Table 1). Average body mass index and heart rate were higher in those with elevated depressive symptoms, and prevalence of hypertension, diabetes, and albuminuria were higher. Both HDL cholesterol and C-reactive protein were higher among participants with elevated depressive symptoms. Similar patterns were observed comparing participants with trait anxiety score $<19$ to those with elevated symptoms. However, the prevalence of albuminuria and average C-reactive protein levels were not significantly different between participants with and without elevated anxiety symptoms.

Figure 1 presents the percentage of participants with $\mathrm{T}$ wave inversions, according to category of anxiety and depressive symptoms. The prevalence of $\mathrm{T}$ wave inversions was lower among those with (1.9\%) compared to those without (2.9\%) elevated anxiety symptoms. However, the prevalence of $\mathrm{T}$ wave inversions was higher among those with elevated depressive symptoms (3.4\%) compared to those without (2.4\%). When participants 
were categorized both according to depressive and anxiety symptoms, the highest prevalence of $\mathrm{T}$ wave inversions was among participants who reported elevated depressive symptoms but who did not report elevated anxiety symptoms. Conversely, the lowest prevalence of $\mathrm{T}$ wave inversions was in participants who reported elevated anxiety but who did not report elevated depressive symptoms.

In logistic regression models that simultaneously included both depressive and anxiety symptoms (Table 2), elevated depressive symptoms were associated with increased odds of $\mathrm{T}$ wave inversions (odds ratio 1.91, 95\% CI 1.30-2.80 in age/sex/race adjusted models), while elevated anxiety score was associated with reduced odds of $\mathrm{T}$ wave inversions (OR $0.56,95 \%$ CI 0.35-0.88). Results were similar after full multivariable adjustment. Variance inflation factors were $\leq .30$, indicating low multi-collinearity between depressive and anxiety symptoms. In gender-stratified analyses, the odds ratios for elevated depressive and anxiety symptoms were consistent for men and women. In subgroups defined by raceethnicity (Table 3), the magnitude of the association between elevated depressive symptoms and $\mathrm{T}$ wave inversions was similar, with odds ratios ranging from 1.80 to 2.80 in multivariable models, although 95\% confidence intervals crossed unity except in AfricanAmericans. In addition, trends remained toward reduced odds of $\mathrm{T}$ wave inversions associated with anxiety, although this was statistically significant only for AfricanAmericans. In sensitivity analyses that included left ventricular mass index by cardiac MRI as an additional covariate $(\mathrm{n}=4295)$, the odds ratios for $\mathrm{T}$ wave inversions were similar to our base case analyses, 2.50 (95\% CI 1.47-4.26, $\mathrm{p}=0.001$ ) for elevated depressive symptoms and 0.38 (95\% CI 0.19-0.74, $\mathrm{p}=0.004)$ for elevated anxiety.

\section{Discussion}

In this sample of men and women without known cardiovascular disease, elevated depressive symptoms were associated with increased odds of ECG T wave inversions in leads other than V1-V3, and elevated trait anxiety symptoms were associated with decreased odds of $\mathrm{T}$ wave inversions. The divergent associations of depressive symptoms and anxiety with $\mathrm{T}$ wave inversions may seem surprising, given their moderate correlation, yet was generally preserved across gender and racial/ethnic subgroups and rigorous sensitivity models. We focused our analyses on primary repolarization abnormalities by excluding participants with prolonged QRS duration. In addition, we excluded participants who reported use of psychoactive medications such as antidepressants and antipsychotics.

Depression is a well-recognized risk factor for cardiovascular mortality among the general population. ${ }^{15}$ Anxiety often co-occurs with depression, and has also been associated with cardiovascular mortality and cardiac events in samples of apparently healthy people. ${ }^{16-20}$ However, large studies that simultaneously assessed depression and anxiety have found that these two conditions may have divergent relations with outcomes. In the population-based Health Study of Nord-Trøndelag County (HUNT-2) involving 61,349 men and women, anxiety symptoms measured by self-report questionnaire were associated with reduced cardiovascular mortality (hazard ratio $0.76,95 \%$ CI $0.69-0.83$ ) and depressive symptoms with increased cardiovascular mortality (hazard ratio $1.23,95 \%$ CI 1.12-1.34) in multivariable models adjusting for both negative emotions. ${ }^{21}$ Also, a study of 5,057 patients 
referred for routine exercise testing found that self-reported anxiety symptoms were associated with lower mortality (hazard ratio $0.81,95 \%$ CI $0.72-0.92$ ) whereas depressive symptoms were associated with increased mortality (hazard ratio $1.14,95 \%$ CI 1.01-1.30). ${ }^{22}$ Cardiac repolarization is a potential avenue for investigation for the differential relations between depression, anxiety, and mortality outcomes that have been observed in previous observational studies.

There are a number of potential important limitations to our study. Of note, the overall proportion of subjects with T wave inversions in MESA (2.6\%) was higher than in the prior study by Aro, et al. $(0.7 \%),{ }^{4}$ although we would speculate that this is the result of the higher average age in MESA (62 years) compared with the latter study (44 years). Despite the attempts to adjust for numerous risk factors in our multivariable analyses, our results may suffer from residual confounding. We used self-reported depressive symptoms and trait anxiety for our indicators of each negative emotion, and these do not substitute for clinical diagnoses of depression or anxiety. Also, our study included only one-time assessments of depressive symptoms and anxiety, and we could not determine whether changes in either symptom affect $\mathrm{T}$ wave amplitude over time. Nonetheless, our analysis opens a potential line of investigation into the differences that have been observed in the relations between depressive symptoms, anxiety, and cardiac prognosis.

\section{Supplementary Material}

Refer to Web version on PubMed Central for supplementary material.

\section{Acknowledgments}

Funding Sources: This research was supported by contracts N01-HC-95159, N01-HC-95160, N01-HC-95161, N01-HC-95162, N01-HC-95163, N01-HC-95164, N01-HC-95165, N01-HC-95166, N01-HC-95167, N01HC-95168 and N01-HC-95169 from the National Heart, Lung, and Blood Institute and by grants UL1-TR-000040 and UL1-TR-001079 from NCRR. The authors thank the other investigators, the staff, and the participants of the MESA study for their valuable contributions. A full list of participating MESA investigators and institutions can be found at http://www.mesa-nhlbi.org. Dr. Whang is supported by a Scientist Development Grant from the AHA Founders Affiliate. Dr. Nazarian is supported by NIH grants K23HL089333 and R01HL116280, and is a PI for research support to Johns Hopkins from Biosense Webster Inc. Dr. Alcantara is supported by NIH grant R01HL115941-01S1. Dr. Shah is supported by NIH grants UL1TR000454 and KL2TR000455. Dr. Davidson is supported by NIH grants HL114924, HL088117 and HL084034.

\section{References}

1. Algra A, Tijssen JG, Roelandt JR, Pool J, Lubsen J. QTc prolongation measured by standard 12-lead electrocardiography is an independent risk factor for sudden death due to cardiac arrest. Circulation. 1991; 83:1888-1894. [PubMed: 2040041]

2. Whang W, Shimbo D, Levitan EB, Newman JD, Rautaharju PM, Davidson KW, Muntner P. Relations between QRS|T angle, cardiac risk factors, and mortality in the third National Health and Nutrition Examination Survey (NHANES III). Am J Cardiol. 2012; 109:981-987. [PubMed: 22221946]

3. Dekker JM, Schouten EG, Klootwijk P, Pool J, Kromhout D. ST segment and T wave characteristics as indicators of coronary heart disease risk: the Zutphen Study. J Am Coll Cardiol. 1995; 25:13211326. [PubMed: 7722128]

4. Aro AL, Anttonen O, Tikkanen JT, Junttila MJ, Kerola T, Rissanen HA, Reunanen A, Huikuri HV. Prevalence and prognostic significance of T-wave inversions in right precordial leads of a 12-lead 
electrocardiogram in the middle-aged subjects. Circulation. 2012; 125:2572-2577. [PubMed: 22576982]

5. Bild DE, Bluemke DA, Burke GL, Detrano R, Diez Roux AV, Folsom AR, Greenland P, Jacob DR Jr, Kronmal R, Liu K, Nelson JC, O'Leary D, Saad MF, Shea S, Szklo M, Tracy RP. Multi-ethnic study of atherosclerosis: objectives and design. Am J Epidemiol. 2002; 156:871-881. [PubMed: 12397006]

6. Bluemke DA, Kronmal RA, Lima JAC, Liu K, Olson J, Burke GL, Folsom AR. The Relationship of Left Ventricular Mass and Geometry to Incident Cardiovascular Events: The MESA (Multi-Ethnic Study of Atherosclerosis) Study. J Am Coll Cardiol. 52:2148-2155. [PubMed: 19095132]

7. DeFilippis AP, Kramer HJ, Katz R, Wong ND, Bertoni AG, Carr J, Budoff MJ, Blumenthal RS, Nasir K. Association Between Coronary Artery Calcification Progression and Microalbuminuria: The MESA Study. JACC: Cardiovascular Imaging. 2010; 3:595-604. [PubMed: 20541715]

8. Natori S, Lai S, Finn JP, Gomes AS, Hundley WG, Jerosch-Herold M, Pearson G, Sinha S, Arai A, Lima JA, Bluemke DA. Cardiovascular function in multi-ethnic study of atherosclerosis: normal values by age, sex, and ethnicity. AJR Am J Roentgenol. 2006; 186:S357-365. [PubMed: 16714609]

9. Radloff LS. The CES-D Scale: A Self-Report Depression Scale for Research in the General Population. Applied Psychological Measurement. 1977; 1:385-401.

10. Spielberger CD. Assessment of state and trait anxiety: Conceptual and methodological issues. Southern Psychologist. 1985; 2:6-16.

11. Davidson KW, Schwartz JE, Kirkland SA, Mostofsky E, Fink D, Guernsey D, Shimbo D. Relation of Inflammation to Depression and Incident Coronary Heart Disease (from the Canadian Nova Scotia Health Survey [NSHS95] Prospective Population Study). Am J Cardiol. 2009; 103:755761. [PubMed: 19268727]

12. Shaffer JA, Epel E, Kang MS, Ye S, Schwartz JE, Davidson KW, Kirkland S, Honig LS, Shimbo D. Depressive symptoms are not associated with leukocyte telomere length: findings from the Nova Scotia Health Survey (NSHS95), a population-based study. PloS one. 2012; 7:e48318. [PubMed: 23133583]

13. Casale PN, Devereux RB, Alonso DR, Campo E, Kligfield P. Improved sex-specific criteria of left ventricular hypertrophy for clinical and computer interpretation of electrocardiograms: validation with autopsy findings. Circulation. 1987; 75:565-572. [PubMed: 2949887]

14. Feldman T, Childers RW, Borow KM, Lang RM, Neumann A. Change in ventricular cavity size: differential effects on QRS and T wave amplitude. Circulation. 1985; 72:495-501. [PubMed: 4017204]

15. Penninx BW, Beekman AT, Honig A, Deeg DJ, Schoevers RA, van Eijk JT, van Tilburg W. Depression and cardiac mortality: results from a community-based longitudinal study. Arch Gen Psychiatry. 2001; 58:221-227. [PubMed: 11231827]

16. Watkins LL, Blumenthal JA, Babyak MA, Davidson JRT, McCants CB Jr, O'Connor C, Sketch $\mathrm{MH}$ Jr. Phobic anxiety and increased risk of mortality in coronary heart disease. Psychosom Med. 2010; 72:664-671. [PubMed: 20639390]

17. Albert CM, Mittleman MA, Chae CU, Lee IM, Hennekens CH, Manson JE. Triggering of sudden death from cardiac causes by vigorous exertion. New England Journal of Medicine. 2000; 343:1355-1361. [PubMed: 11070099]

18. Rutledge T, Kenkre TS, Bittner V, Krantz DS, Thompson DV, Linke SE, Eastwood JA, Eteiba W, Cornell CE, Vaccarino V, Pepine CJ, Johnson BD, Bairey Merz CN. Anxiety associations with cardiac symptoms, angiographic disease severity, and healthcare utilization: the NHLBI-sponsored Women's Ischemia Syndrome Evaluation. Int J Cardiol. 2013; 168:2335-2340. [PubMed: 23410495]

19. Roest AM, Martens EJ, de Jonge P, Denollet J. Anxiety and Risk of Incident Coronary Heart Disease: A Meta-Analysis. J Am Coll Cardiol. 2010; 56:38-46. [PubMed: 20620715]

20. Kawachi I, Colditz GA, Ascherio A, Rimm EB, Giovannucci E, Stampfer MJ, Willett WC. Prospective study of phobic anxiety and risk of coronary heart disease in men. Circulation. 1994; 89:1992-1997. [PubMed: 8181122] 
21. Mykletun A, Bjerkeset O, Dewey M, Prince M, Overland S, Stewart R. Anxiety, depression, and cause-specific mortality: the HUNT study. Psychosom Med. 2007; 69:323-331. [PubMed: 17470669]

22. Herrmann C, Brand-Driehorst S, Buss U, Ruger U. Effects of anxiety and depression on 5-year mortality in 5,057 patients referred for exercise testing. J Psychosom Res. 2000; 48:455-462. [PubMed: 10880666] 


\section{Highlights}

1. We examined the relation of abnormal repolarization to depressive and anxiety symptoms.

2. Elevated depressive symptoms were associated with increased odds of $\mathrm{T}$ wave inversions.

3. Greater trait anxiety was associated with a reduced odds of $\mathrm{T}$ wave inversions.

4. Negative emotions may have unique relations with cardiac repolarization. 


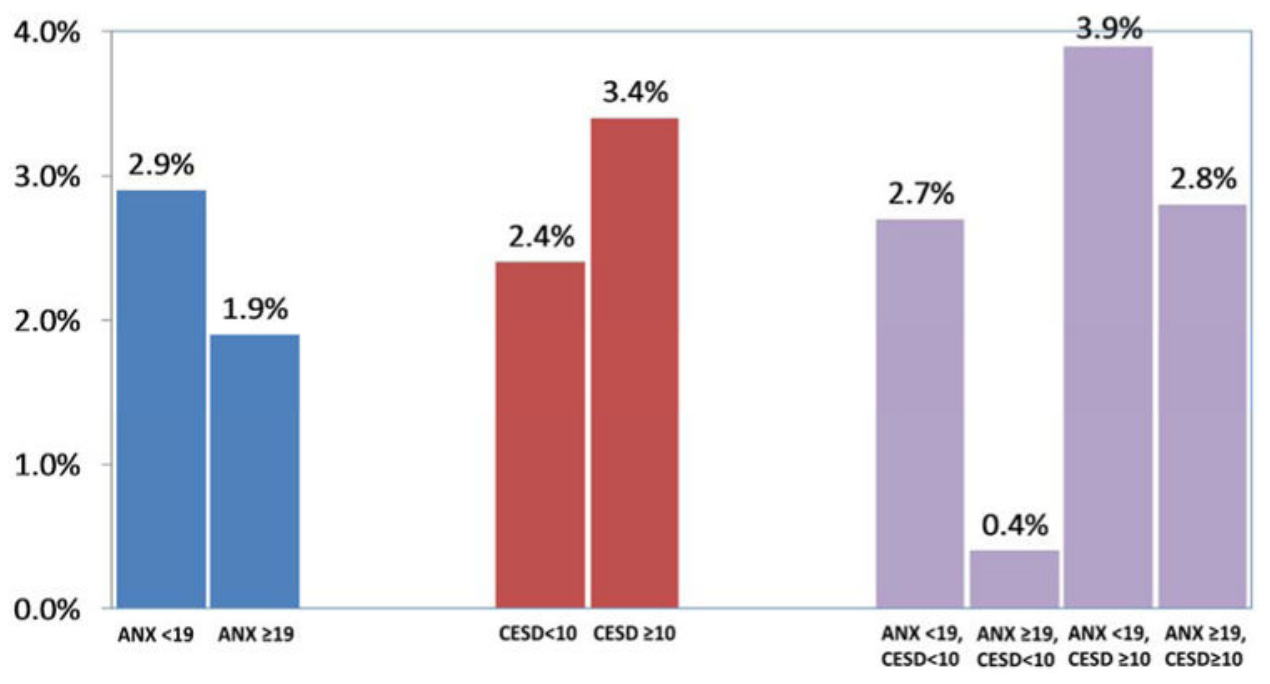

Figure 1.

Prevalence of electrocardiographic $\mathrm{T}$ wave inversions according to elevated depressive and anxiety symptoms, defined by upper quartile of CES-D (Center for Epidemiologic StudiesDepression) or ANX (Spielberger trait anxiety) score. 


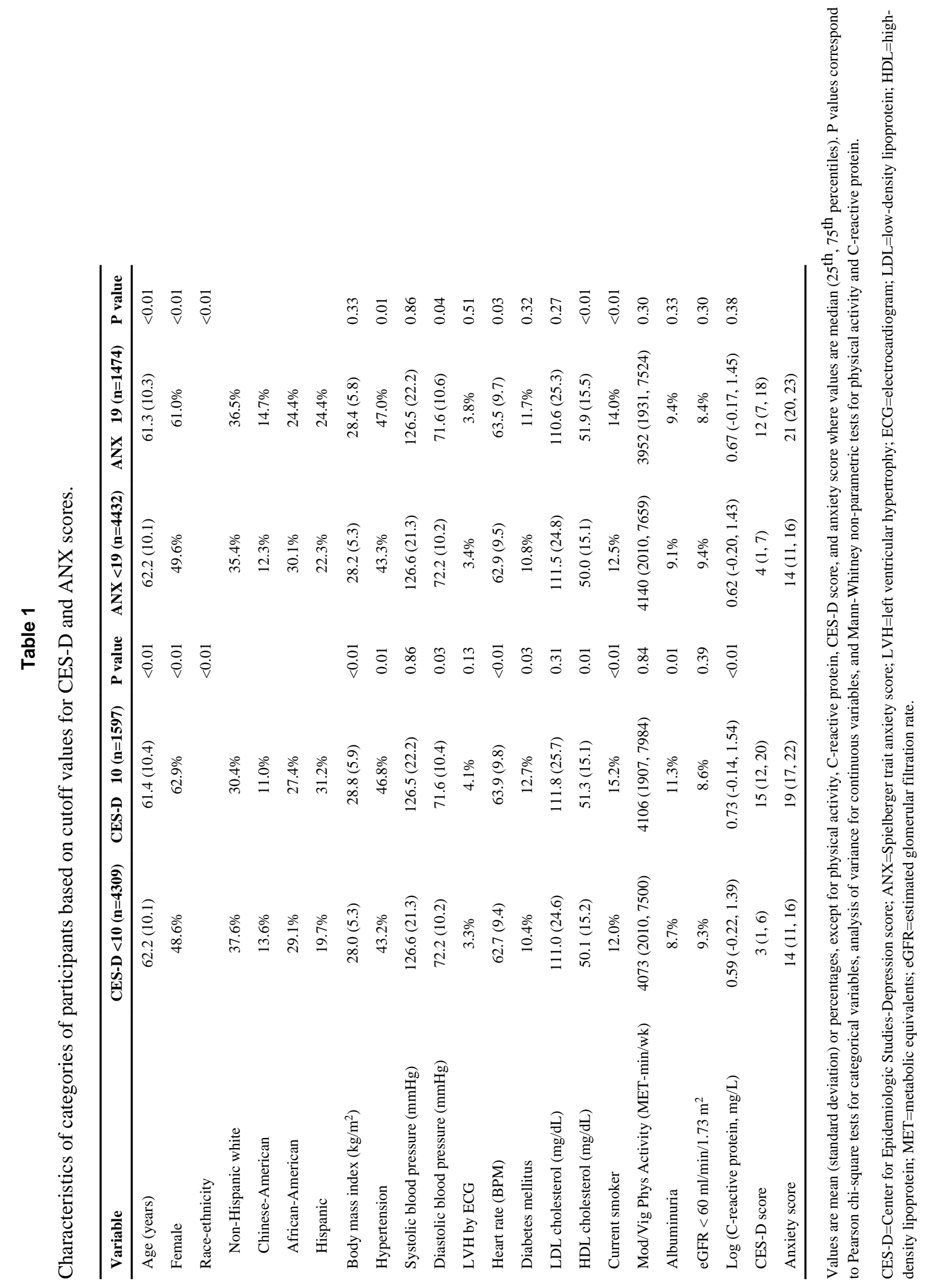




\section{Table 2}

Logistic regression models of $\mathrm{T}$ wave inversions, in all subjects combined and stratified by gender.

\begin{tabular}{lcccc}
\hline Grouping & Age/race +/- sex adjusted & P value & Multivariable & P value \\
\hline All $(n=5906)$ & & & & \\
CES-D $\geq 10$ & $1.91(1.30,2.80)$ & 0.001 & $2.02(1.33,3.06)$ & 0.001 \\
ANX $\geq 19$ & $0.56(0.35,0.88)$ & 0.013 & $0.47(0.29,0.77)$ & 0.003 \\
Men $(n=2807)$ & & & & \\
CES-D $\geq 10$ & $2.00(1.15,3.47)$ & 0.014 & $2.00(1.08,3.69)$ & 0.027 \\
ANX $\geq 19$ & $0.60(0.30,1.18)$ & 0.135 & $0.43(0.20,0.93)$ & 0.031 \\
Women $(n=3099)$ & & & & \\
CES-D $\geq 10$ & $1.79(1.04,3.06)$ & 0.035 & $1.77(0.98,3.21)$ & 0.059 \\
ANX $\geq 19$ & $0.55(0.29,1.04)$ & 0.064 & $0.55(0.28,1.10)$ & 0.093 \\
\hline
\end{tabular}

All models adjust simultaneously both for CES-D and ANX score. In addition to age/race, multivariable models also adjusted for center, body mass index, hypertension, diabetes mellitus, low-density lipoprotein and high-density lipoprotein cholesterol levels, systolic and diastolic blood pressure, cigarette smoking (never, former, or current), self-reported moderate and vigorous physical activity in quartiles, estimated glomerular filtration rate $<60$, albuminuria, left ventricular hypertrophy by Cornell ECG criteria, log-transformed highly-sensitive plasma C-reactive protein levels, benzodiazepine, and beta blocker use. Additionally, models with men and women combined adjusted for gender. Due to missing data, sample sizes were reduced in multivariable models ( $n=5831$ for all participants, $n=2776$ for men, $n=3055$ for women). 


\section{Table 3}

Logistic regression models of $\mathrm{T}$ wave inversions, stratified by race-ethnicity.

\begin{tabular}{lcccc}
\hline Grouping & Age/sex adjusted & P value & Multivariable & P value \\
\hline White $(n=2105)$ & & & & \\
CES-D $\geq 10$ & $1.79(0.65,4.92)$ & 0.259 & $1.80(0.61,5.26)$ & 0.285 \\
ANX $\geq 19$ & $0.57(0.19,1.78)$ & 0.336 & $0.52(0.17,1.67)$ & 0.273 \\
Chinese-American $(n=761)$ & & & & \\
CES-D $\geq 10$ & $1.78(0.50,6.41)$ & 0.377 & $2.80(0.47,16.54)$ & 0.256 \\
ANX $\geq 19$ & $0.93(0.24,3.65)$ & 0.913 & $0.79(0.12,5.21)$ & 0.809 \\
African-American $(n=1693)$ & & & & \\
CES-D $\geq 10$ & $2.09(1.23,3.56)$ & 0.006 & $1.95(1.09,3.51)$ & 0.025 \\
ANX $\geq 19$ & $0.44(0.22,0.88)$ & 0.021 & $0.37(0.17,0.80)$ & 0.011 \\
Hispanic $(n=1347)$ & & & & \\
CES-D $\geq 10$ & $1.60(0.72,3.57)$ & 0.250 & $2.03(0.80,5.13)$ & 0.134 \\
ANX $\geq 19$ & $0.78(0.31,1.94)$ & 0.593 & $0.62(0.21,1.78)$ & 0.373 \\
\hline
\end{tabular}

$*$

* All models adjust simultaneously both for CES-D and ANX score. In addition to age/gender, multivariable models also adjusted for center, body mass index, hypertension, diabetes mellitus, low-density lipoprotein and high-density lipoprotein cholesterol levels, systolic and diastolic blood pressure, cigarette smoking (never, former, or current), self-reported moderate and vigorous physical activity in quartiles, estimated glomerular filtration rate $<60$, albuminuria, left ventricular hypertrophy by Cornell ECG criteria, log-transformed highly-sensitive plasma C-reactive protein levels, benzodiazepine, and beta blocker use. 